http://jmscr.igmpublication.org/home/ ISSN (e)-2347-176x ISSN (p) 2455-0450 crossref DOI: https://dx.doi.org/10.18535/jmscr/v8i6.84

\title{
Small Incision Cataract Surgery (A Safe, Cheap and Affordable Alternative to Phacoemulsification) (Let's make it popular)
}

\author{
Authors \\ Samar H Shoeir, FICO, MRCS Ed, MSc ${ }^{1 *}$, Sanaa A Mohamed, MD, MSc ${ }^{2}$ \\ Doaa A Hussien FICO, MRCSEd ${ }^{3}$ \\ ${ }^{1}$ Cataract and Glaucoma Specialist, Al Fayoum Ophthalmology Hospital \\ ${ }^{2}$ Lecturer of Ophthalmology, Azhar University \\ ${ }^{3}$ Ophthalmology Specialist, Benisuef Ophthalmology Hospital \\ *Corresponding Author \\ Samar H Shoeir, FICO, MRCS Ed, MSc
}

\begin{abstract}
Purpose: To study the effectiveness and adverse events associated with the surgical treatment of cataracts; focusing on SICS as treatment option to make recommendations on the most suitable treatment option for cataract in outreach.

Settings: Senoris general hospital (Al Fayoum city).

Design: This prospective cross-sectional study.

Methods: This study enrolled patients who underwent MSICS between April 2018 to April 14 2019. The primary outcome measure was post-operative, pinhole corrected VA on day 1 and 4 weeks. The secondary outcomes were complications as well as the costs of surgery.

Results: A total number of 120 patients, 45 of them were males (37.5\%), mean age of $62.0917 \pm 9.7$ years underwent MSICS. On post-operative day one, 70 eyes (58.3\%) had good visual acuity (6/6 to 6/18), 50 eyes $(41.6 \%)$ borderline $(<6 / 18$ to $6 / 60)$ and $0(0 \%)$ had poor outcome less than $6 / 60$.

At 4 weeks postoperatively 120 eyes had good vision.

Among all operated eyes, 32 eyes (26.6\%) had intra-operative complications. The most common intraoperative complication was premature entry in 8 eyes $(6.7 \%)$. The frequent complication during the immediate postoperative period was corneal edema (34.1\%). It was resolved within 2 weeks with good VA. The total surgical costs per case were estimated at (400 LE) (\$25).

Conclusion: Manual SICS is significantly faster and less technology-dependent than phacoemulsification, it may be a more appropriate cataract surgery in outreach.

Keywords: Cataract, Manual small incision cataract surgery.
\end{abstract}

\section{Introduction}

Cataract, one of the most common eye diseases ${ }^{1}$ and leading cause of preventable blindness worldwide $^{2}$.
In most developing countries, blindness is associated with considerable economic and social implications which impacts on the current difficulties of vulnerable populations who reside in under-served areas ${ }^{3}$. 
The majority of people who are affected with cataracts reside in developing countries; these areas have limited eye care capabilities to cope with the high demand for cataract surgery, limited capacity, infrastructure and technology to care for the visually impaired ${ }^{4}$.

Cataracts are treatable through cataract surgery, the most common procedure performed in ophthalmology through the surgical removal of the opacified lens ${ }^{5}$ facilitated by the implantation of an intraocular lens (IOL) ${ }^{6}$.

To overcome the burden of cataract blindness, there must be sufficient surgical coverage and good surgical outcomes, safety, early visual rehabilitation and postoperative emmetropia.

Small-incision cataract surgery (SICS) is also called manual small-incision cataract surgery (MSICS) or suture less extra-capsular cataract extraction (SECCE). It was developed in the United States and Israel and made popular in India, with the large proportion of surgeries undertaken ${ }^{7}$. In this technique, extra capsular extraction is performed. The nucleus is prolapsed and removed through a $(6 \mathrm{~mm})$ scleral tunnel and aspirates the remaining cortex ${ }^{8}$. A single-piece rigid IOL (poly methyl methacrylate) with a 6.0 $\mathrm{mm}$ optic is then implanted in the capsular bag and the anterior chamber pressurized. The tunnel is self-sealing and the wound does not need sutures in most cases ${ }^{9}$. It is a safe, cost-effective procedure with very good outcomes. The technique is well described in the literature, as is the management of its complications.

The quality of SICS, and most importantly the outcome for the patient, can be excellent. The World Health Organization (WHO) advises aiming for post-operative outcomes of at least $80 \%$ good presenting vision or at least $90 \%$ with best-corrected vision, and this is attainable with SICS.

\section{Material and Methods}

This prospective cross-sectional study enrolled patients who underwent routine manual small incision cataract surgery between April 2018 to
April 14 2019. The study was conducted in accordance with the tenets of the Helsinki declaration. A written informed consent was obtained.

Exclusion criteria were patients with traumatic, uveitic or developmental cataract.

Preoperatively all patients underwent full ophthalmological examination (Best corrected visual acuity, schiotz tonometry, fundus examination if possible and B scan ultrasonography in patients with dense media opacity).

Intra-ocular lens power was calculated in all settings using manual keratometer and contact Ascan biometry.

Cataract surgery was done using manual small incision surgical technique with a superior approach under peri-bulbar anesthesia. Single piece polymethyl methacrylate intraocular lenses (PMMA IOL) were inserted in all patients with no sutures were placed in uncomplicated cases and all were given a sub-conjunctival injection with a combination of gentamycin and dexamethasone.

All patients received the same postoperative combination of Moxifloxacin and Prednisolone (1\%) eye drops five times per day from day 1 till day 8, 4 times per day in the 2 nd week, and gradually tapering for another 4 weeks. The ophthalmologist examined all patients on postoperative day 1 and after 2 to 4 weeks with a slit-lamp.

The primary outcome measure was post-operative, pinhole corrected VA on day 1 and 4 weeks. The secondary outcomes were intra and post-operative complications as well as the overall costs of cataract surgery considering both patients and eye care programs.

Visual acuity (VA) was categorized using the WHO guidelines: good $6 / 6$ to $6 / 18$, borderline $6 / 18$ to $6 / 60$, and poor less than $6 / 60$. Preoperative VA was best corrected by using patient refraction. All the post-operative VA on day one and at 4 weeks was pin hole corrected.

Patient cost estimates included pre, intra and postoperative supplies needed for the patient and were 
based on government local currency (Egyptian Pound) and American dollar.

\section{Statistical Analysis}

Data were collected, coded, revised and entered to the Statistical Package for

Social Science (IBM SPSS) version 20. The data were presented as number and

Percentages for the qualitative data, mean, standard deviations and ranges for thequantitative data with parametric distribution and median with inter quartile range (IQR) for the quantitative data with non-parametric distribution.

The confidence interval was set to $95 \%$ and the margin of error accepted was

Set to $5 \%$. So, the p-value was considered significant as the following:

$\mathrm{P}>0.05$ : Non significant (NS)

$\mathrm{P}<0.05$ : Significant $(\mathrm{S})$

$\mathrm{P}<0.01$ : Highly significant (HS)

\section{Results}

Table 1: Sex Distribution in the study

\begin{tabular}{|c|c|c|c|c|}
\hline \multicolumn{2}{|l|}{ Gender } & Frequency & Percent & Valid Percent \\
\hline \multirow{3}{*}{} & $\mathrm{F}$ & 75 & 62.5 & 62.5 \\
\cline { 2 - 5 } & $\mathrm{M}$ & 45 & 37.5 & 37.5 \\
\cline { 2 - 5 } & Total & 120 & 100.0 & 100.0 \\
\hline
\end{tabular}

Table 2: Preoperative BCVA

\begin{tabular}{|l|c|c|}
\hline Preoperative BCVA & frequency & Percent \\
\hline HM & 16 & 13.3 \\
\hline CF $10-25 \mathrm{~cm}$ & 6 & 4.9 \\
\hline $1 / 60$ & 4 & 3.3 \\
\hline $2 / 60$ & 19 & 15.8 \\
\hline $3 / 60$ & 12 & 10 \\
\hline $4 / 60$ & 11 & 9.2 \\
\hline $5 / 60$ & 16 & 13.3 \\
\hline $6 / 60$ & 18 & 15.0 \\
\hline $6 / 36$ & 17 & 14.2 \\
\hline Total & 120 & $100 \%$ \\
\hline
\end{tabular}

Table 3: First Day Postoperative Visual Acuity

\begin{tabular}{|c|c|c|c|}
\hline \multicolumn{2}{|l|}{ First Day VA } & Frequency & Percent \\
\hline \multirow{6}{*}{} & $6 \backslash 12$ & 12 & 10.0 \\
\cline { 2 - 4 } & $6 \backslash 18$ & 58 & 48.3 \\
\cline { 2 - 4 } & $6 \backslash 24$ & 22 & 18.3 \\
\hline & $6 \backslash 26$ & 1 & .8 \\
\hline & & & \\
\cline { 2 - 4 } & 6136 & 23 & 19.2 \\
\cline { 2 - 4 } & $6 \backslash 60$ & 4 & 3.3 \\
\cline { 2 - 4 } & Total & 120 & 100.0 \\
\hline
\end{tabular}

Table 4: 4 Weeks Postoperative Visual Acuity

\begin{tabular}{|c|c|c|c|}
\hline \multicolumn{2}{|c|}{ Four Weeks VA } & Frequency & Percent \\
\hline \multirow{5}{*}{} & $6 \backslash 12$ & 46 & 38.3 \\
\cline { 2 - 4 } & $6 \backslash 18$ & 10 & 8.3 \\
\cline { 2 - 4 } & 616 & 27 & 22.5 \\
\cline { 2 - 4 } & $6 \backslash 9$ & 37 & 30.8 \\
\cline { 2 - 4 } & Total & 120 & 100.0 \\
\hline
\end{tabular}

Table 5: Early postoperative complications

\begin{tabular}{|c|c|c|c|}
\hline \multicolumn{2}{|c|}{ Postoperative Complications } & Frequency & Percent \\
\hline \multirow{7}{*}{} & Striate keratopathy & 4 & 3.3 \\
\cline { 2 - 4 } & Corneal edema & 41 & 34.1 \\
\cline { 2 - 4 } & Total & 120 & 100.0 \\
\hline
\end{tabular}

\section{Results}

Manual small incision cataract surgery was performed on 120 patients, 45 of them were males (37.5\%) and 75 were females $(62.5 \%)$; mean age of $62.0917 \pm 9.7$ years (range 41 to 80 years) (Table 1).

The majority of eyes had immature corticonuclear and posterior subcapsular $(83.1 \%)$ or mature cataract (16.9\%).

Preoperatively 46 eyes $(38.1 \%)$ had VA $<3 / 60$ and 39 eyes (32.5\%) had VA from $3 / 60$ to $<6 / 60$ 35 eyes (29.2\%) had VA from 6/60 to 6/18 (Table 2).

On post-operative day one, 70 eyes $(58.3 \%)$ had good visual acuity $(6 / 6$ to $6 / 18), 50$ eyes $(41.6 \%)$ borderline $(<6 / 18$ to $6 / 60)$ and $0(0 \%)$ had poor outcome less than 6/60 (Table 3).

At 4 weeks postoperatively 120 eyes (100\%) eyes had good, $0(0 \%)$ borderlines and $0(0 \%)$ had poor VA (Table 4).

Among all Operated Eyes, 32 eyes (26.6\%) had intra-operative complications. Posterior capsular rupture did not occur in our sample.

The most common intra-operative complication was premature entry in 8 eyes $(6.7 \%)$. The frequent complications during the immediate postoperative period were striate keratopathy (3.3\%), mild corneal edema (34.1\%). Most of corneal edema and striate keratopathy were resolved within 2-3 weeks with good VA. Eight eyes had sclero-corneal tunnel suturing for wound leak and 5 needed hypertonic saline added postoperatively for corneal edema. 
At 4 wks. Follow up none of the eyes needed additional treatment. There were no cases of endophthalmitis in our cases (Table 5).

The total surgical costs per case were estimated at (400 LE) (\$25).

\section{Discussion}

In our study, pinhole corrected VA $\geq 6 / 18$ in the first postoperative day was $(58.3 \%)$ and raised to $100 \%$ at 4 week postoperatively.

A similar study in Nepal reported that pinhole corrected VA $\geq 6 / 18$ remained the same at day one and $2-3$ weeks, $\left(79.7 \%\right.$ and $79.1 \%$ respectively) ${ }^{10}$ , another study reported that the uncorrected VA $\geq$ $6 / 18$ declined from $76.8 \%$ day one post-operative to $70.5 \%$ at $4-6$ weeks $^{11}$. The reasons for decreasing VA were uncorrected refractive error and surgically induced astigmatism.

Zia et al. (2010) also reported similar results in his study on patients underwent SICS with visual acuity $6 / 18$ or better was recorded $(43.33 \%)^{12}$.

An Indian study reported that best corrected VA $>6 / 18$ at about 6 weeks after surgery was $(82.7 \%)$ 13 .

This Gender Disparity underlines the need to provide more active outreach activities to reduce the financial and cultural barriers faced by women by increasing activities involve working with mother's group leaders and female community health volunteers.

A total of $26.6 \%$ of patients had intra-operative complications in our study with premature entry being the most common $(6.7 \%)$. All of these patients had good final pinhole VA at 4 weeks postoperatively which were similar to a Nepalian study with A total of $6 \%$ of patients had intraoperative complications with premature entry being the most common $(2.1 \%)^{11 .}$

Another Pakistani study ${ }^{14}$ showed that $7.8 \%$ had intraoperative or immediate post-operative complications with hyphaema $(4.2 \%)$ is the most common.

In our study posterior capsule rupture didn't occur $(0 \%)$, but was observed in 7 eyes $(0.7 \%)$ in a study conducted in Nepal in $2017^{11}$. The likely reasons include inadequate operation theatre setup, poorly illuminated portable microscope and lack of microsurgical facilities as reported by the surgeons in the study.

Another study reported reported that (5.8\%) had intraoperative complications, with posterior capsular rupture $(3.9 \%)$ was the most common (Limbu et al) ${ }^{16}$. Studies in India and Nepal reported higher incidence of posterior capsular rupture $(1.9 \%, 3.63 \%)$ and $(20.5 \%)$ than our study $14,16,17$.

In our study, $15 \%$ eyes had postoperative complication on day one $(37.4 \%)$, striate keratopathy and mild corneal edema (3.3\%, $34.1 \%)$ respectively. None of the post-operative complications were associated with poor visual acuity at 4 weeks follow-up. Four studies reported lower post operative complications rate of $15 \%$, $9.3 \%, 9.3 \%$ and $6.2 \%$ respectively ${ }^{9,10,11,15}$. Higher post-operative complications in our studies were probably due to in appropriate use of viscoelastic and learning curve of the operating surgeon.

In our study the total surgical costs per case were estimated at (USD 25). In Gogate et al $^{7}$ study with average cost of SICS surgery was (USD15.34).

\section{Limitations of the study}

The limitation of the study was inability to detect late post operative complications like surgically induced astigmatism and posterior capsule opacification due to short follow up period.

\section{What Was Known}

In outreach and remote areas in Egypt small incision cataract surgery is not popular and the main technique for cataract extraction was extra capsular cataract extraction (ECCE) technique which has several disadvantages including high postoperative cylinder and poor patient satisfaction especially in early post-operative period

\section{What This Paper Adds}

Manual small incision cataract surgery (MSICS) can be easily performed in remote areas where 
technology is not available and leaving high patient satisfaction with good visual outcome.

\section{Conclusion}

Despite the advantages of Phaco, the technique also has some weaknesses. The Phaco equipment requires access to biomedical engineers to maintain the equipment which is another cost factor18.

Manual SICS is significantly faster, less expensive, and less technology-dependent than phacoemulsification, it may be a more appropriates chnique in eyes with mature cataract in the developing world.

\section{Financial Interests: None \\ Financial Support: None}

\section{References}

1. LuiB, XuL, Ya Xing W, and Jonas JB. Prevalence of Cataract Surgery and Postoperative Visual Outcome in Greater Beijing: The Beijing Eye Study. American Academy of Ophthalmology. 2009. Doi:10.1016/j.ophtha.

2. YorstonD, Gichuhi S, Wood M, and Foster A. Does prospective monitoring improves cataract surgery outcome in Africa? British Journal of Ophthalmology.2002; 86:543547.

3. Komolafe OO, Ashaye AO, Ajayi BGK and Bekibele CO. Visual Impairment from Age-Related.

4. Cataract among an Indigenous African Population. Eye.2010; 24:53-58.

5. Tabin G, Chen $M$, and Espandar L. Cataract surgery for the developing world. Current Opinionin Ophthalmology, 2008; 19:55-59.

6. Schwiegerling. J. Intraocular Lenses. Department of Ophthalmology. University of Arizona, Arizona. Available from: http://www.mhprofessional.com/handbook ofoptics/pdf/Handbook_of_Optics_vol3_c h2 1.pdf [Accessed02March2012].
7. Malik PK, Keshri PK, Pathak A, Yadhuvanshi HK, and Kapoor P. Cataract Blindness in Developing CountriesProcedure of choice for a Large Population, Asian Journal of Ophthalmology.2002;4(1):9-11.

8. Gogate P. Comparison of various techniques for cataract surgery, their efficacy, safety and cost. Oman Journal of Ophthalmology.2010; 3(3):105-106.

9. Health Care. Phacoemulsification. Available from: http://health.indiamart.com/eyecare [Accessedon22August 2011].

10. Venkatesh R, Tan SH Colin, Sengupta S, Ravindran RD, Krishnan KT, and Chang DF. Phacoemulsification versus manual small-incision cataract surgery for white cataract. J. Cataract Refract. Surg.2010; 36:1849-1854.

11. Hennig A, Kumar J, Yorston D and Foster A. Sutureless cataract surgery with nucleus extraction: Outcome of a prospective study in Nepal. Br J Ophthalmol 2003; 87:266270.

12. Laxmi Devi Manandhar1, Salma KC Rai2, Kabindra Bajracharya3, Ram Prasad Kandel4, Prasanna Sharma5, Ken Bassett6-Asian Journal of Medical Sciences | Jul-Aug 2018 | Vol 9 | Issue 4.

13. Zia S, Raza, and Syed IA. Comparison of Small Incision Cataract Surgery with Extra Capsular Cataract Extraction. Journal of Rawalpindi Medical College (JRMC). 2010;14(2):84-86.

14. Gogate P and Kulkarni AN. Comparison of Cataract Surgery in a Base Hospital and in Peripheral Eye Camps. Community Eye Health 2002; 15:26-27.

15. Khan MT, Jan S, Hussain Z, Karim S, Khalid MK and Mohammad L. Visual Outcome and Complications of Manual Sutureless Small Incision Cataract Surgery. Pak J Ophthalmol 2010;26:32-38 
16. Limbu B and Jha HC. Intraoperative Complications of High Volume Sutureless Cataract Surgery in Nepal: A Prospective Study. Kathmandu Univ Med J 2014; 47:194-197.

17. Hosamani S, Vallabha K and Warad V. Post-operative complications and visual outcome in eye camp patients undergoing sutureless cataract surgery at a Base Hospital in Vijayapura District, South India. Niger J Ophthalmol 2015; 23:16-21.

18. Shrestha JK, Pradhan YM and Snellingen T. Outcomes of extracapsular surgery in eye camps of eastern Nepal. $\mathrm{Br} \mathbf{J}$ Ophthalmol 2001; 85:648-652.

19. Boughton B. Phacoand ECCE: Comparing the Costs and Benefits, EYENET: 2009; 43-47. 